\title{
ASSESSMENT OF THE ORION-SLS INTERFACE MANAGEMENT PROCESS IN ACHIEVING THE EIA 731.1 SYSTEMS ENGINEERING CAPABILITY MODEL GENERIC PRACTICES LEVEL 3 CRITERIA
}

\author{
John J. Jellicorse ${ }^{1}$ \\ NASA Marshall Space Flight Center, Huntsville, Alabama, 39529 \\ Shamim A. Rahman ${ }^{2}$ \\ NASA Johnson Space Center, Houston, Texas, 77059
}

\begin{abstract}
NASA is currently developing the next generation crewed spacecraft and launch vehicle for exploration beyond earth orbit including returning to the Moon and making the transit to Mars. Managing the design integration of major hardware elements of a space transportation system is critical for overcoming both the technical and programmatic challenges in taking a complex system from concept to space operations. An established method of accomplishing this is formal interface management. In this paper we set forth an argument that the interface management process implemented by NASA between the Orion Multi-Purpose Crew Vehicle (MPCV) and the Space Launch System (SLS) achieves the Level 3 tier of the EIA 731.1 System Engineering Capability Model (SECM) for Generic Practices. We describe the relevant NASA systems and associated organizations, and define the EIA SECM Level 3 Generic Practices. We then provide evidence for our compliance with those practices. This evidence includes discussions of: NASA Systems Engineering Interface (SE) Management standard process and best practices; the tailoring of that process for implementation on the Orion to SLS interface; changes made over time to improve the tailored process, and; the opportunities to take the resulting lessons learned and propose improvements to our institutional processes and best practices. We compare this evidence against the practices to form the rationale for the declared SECM maturity level.
\end{abstract}

$\begin{array}{ll}B E O & =\text { Beyond Earth Orbit } \\ C \& D H & =\text { Command and Data Handling } \\ C M & =\text { Crew Module } \\ C P I T & =\text { Cross Program Integration Team } \\ C R & =\text { Change Request } \\ C S I & =\text { Cross Systems Integration } \\ E S D & =\text { Exploration Systems Development } \\ E I A & =\text { Electronic Industries Association } \\ F A & =\text { Functional Area } \\ G S D O & =\text { Ground Systems Development and Operations } \\ G P & =\text { Generic Practices } \\ I C D & =\text { Interface Control Document } \\ I R D & =\text { Interface Requirements Document }\end{array}$

\footnotetext{
${ }^{1}$ Co-Lead for Orion-SLS Interfaces Working Group, SLS Program support, Cross-Program Integration Team, and AIAA Member Grade for first author.

${ }^{2}$ Co-Lead for Orion-SLS Interfaces Working Group, Orion Program support, Cross-Program Integration Team, NASA JSC Engineering Directorate, and AIAA Associate Fellow, and AIAA Board of Directors 2010-2016.
} 


$\begin{array}{ll}I S P E & =\text { Integrated Spacecraft and Payload Element } \\ I T L & =\text { Integrated Test lab } \\ I T T & =\text { Integration Task Team } \\ H E O & =\text { Human Exploration and Operations } \\ J I C B & =\text { Joint Integration Control Board } \\ J P C B & =\text { Joint Program Control Board } \\ J I V & =\text { Joint Interface Verification } \\ J S C & =\text { Johnson Space Center } \\ K S C & =\text { Kennedy Space Center } \\ L A S & =\text { Launch Abort System } \\ M B S E & =\text { Model Based Systems Engineering } \\ M P C V & =\text { Multi-Purpose Crew Vehicle } \\ M S A & =\text { MPCV Spacecraft Adapter } \\ M S F C & =\text { Marshall Space Flight Center } \\ N P R & =\text { NASA Procedural Requirements } \\ O S I W G & =\text { Orion SLS Interface Working Group } \\ S A & =\text { Spacecraft Adapter } \\ \text { SE } & =\text { Systems Engineering } \\ S E C M & =\text { Systems Engineering Capability Model } \\ S I L & =\text { Systems Integration Lab } \\ S L S & =\text { Space Launch System } \\ S M & =\text { Service Module } \\ S P & =\text { Specific Practice } \\ S P I E & =\text { Spacecraft and Payload Integration and Evolution } \\ S R M & =\text { Solid Rocket Motor } \\ T C M & =\text { Technical Coordination Meeting } \\ T I M & =\text { Technical Interchange Meeting } \\ V C R M & =\text { Verification Cross Reference Matrix } \\ & \end{array}$

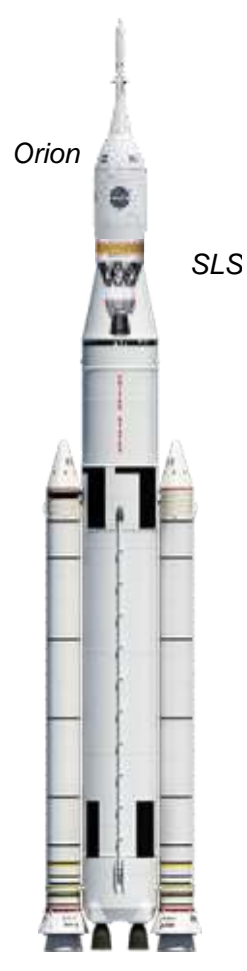

Figure 1. EM-1 stack.

\section{Introduction}

$\mathrm{N}$ ASA is developing the next generation human-rated spacecraft and launch vehicle for exploration beyond earth orbit (BEO) including returning to the Moon and making the transit journey to Mars. Managing the integration of major hardware elements of a space transportation system (see Figure 1) is critical for overcoming both the technical and programmatic challenges in evolving a complex system from concept to space operations. Formal interface management is an established and rigorous process available to help accomplish this.

The importance of technical interfaces is a sine qua non in the engineering of complex systems in aerospace. As stated by Price $^{1}$ (in an equivalent aviation context):

The identification and management of system interactions forms a major part of the systems engineering practices for system architecture, but methodologies for effective identification and management of interactions arising as a result of technical interfaces are still problematic.

The present paper supplies a particular situation and a particular approach to interface management as undertaken for interfacing between two major NASA program entities, and their associate technical hardware. Adding to Price's words, this activity is another attempt to explore and learn how to manage interfaces, where there is unlikely to be a well-established approach and precedent. The interest in finding leaner ways to effectively manage complex interfaces further motivates trying new approaches such as the one to be described.

In this paper we set forth an argument that the interface management process, implemented by NASA between the Orion MPCV and the SLS, has achieved Level 3 for Generic Practices in the EIA 731.1 System Engineering Capability Model $^{2}$. First, as background, we will describe the relevant space systems and associated development organizations and then provide an overview of the EIA SECM and detail the Level 3 Generic Practices. Next, we will provide evidence of our compliance with the SECM Level 3 Generic Practices. This will include a discussion of: 1) NASA SE Interface management standard process and best practices, 2) the tailoring of those processes for implementation on the Orion to SLS interface, 3) what we have modified over time to improve our tailored process, and, 4) our plans to take the lessons learned and to propose improvements to our institutional processes and best practices. Finally, we

American Institute of Aeronautics and Astronautics 
will compare this evidence against the criteria to form the rationale for the declared SECM Generic Practices maturity level.

\section{NASA Context}

\section{A. Orion and SLS Vehicle Architecture}

\section{Vehicle Definition}

The Orion MPCV is the spacecraft being developed by NASA to transfer flight crews, research cargo, and support equipment from Earth to cislunar space, and subsequently return the crew to Earth's surface for the initial exploration mission (EM-1). The essentials of the overall vehicle and its maturity is summarized by $\mathrm{Creech}^{3}$ (2014), and more recently by Donohue ${ }^{4}$ (2016). The Orion System consists of a Crew Module (CM), Service Module (SM), Launch Abort System (LAS), and Spacecraft Adapter (SA). The essentials of the overall vehicle and its maturity is summarized by Marshall et al..$^{5}$ (2013), and more recently by Norris et al. ${ }^{6}(2016)$.

The SLS is a heavy-lift vehicle that supports efficient and affordable launch missions in support of human exploration beyond earth orbit. The SLS Block 1 configuration consists of a Core Stage using four RS-25 engines, augmented by two 5-segment solid rocket motors (SRM) as the first stage, and an Integrated Spacecraft and Payload Element (ISPE) based on a Delta-IV derived interim cryogenic propulsion stage (ICPS) as the second stage. The SLS is used on EM-1 to place the Orion spacecraft into a Trans Lunar Injection (TLI) trajectory.

\section{Interface Definition}

The functional interfaces between the two systems and their associated elements are shown in Figure 2. The mechanical and environmental interfaces are between the Orion SA and the SLS ISPE elements. The Command \& Data Handling (C\&DH) interfaces (discrete signals and digital information) are between the Orion CM and both the SLS Core Stage and the ISPE Elements, since the SLS stages have independent flight computers. The schematic illustrates using arrows, the directionality of influence between Orion and SLS elements. For instance, mechanical interfaces influence both sides, whereas a discrete signal is sent only in one direction. Each entity and arrow in the figure constitutes an interface consideration, or constraint, or design driving function that is given importance through one or more groups of specified requirements.

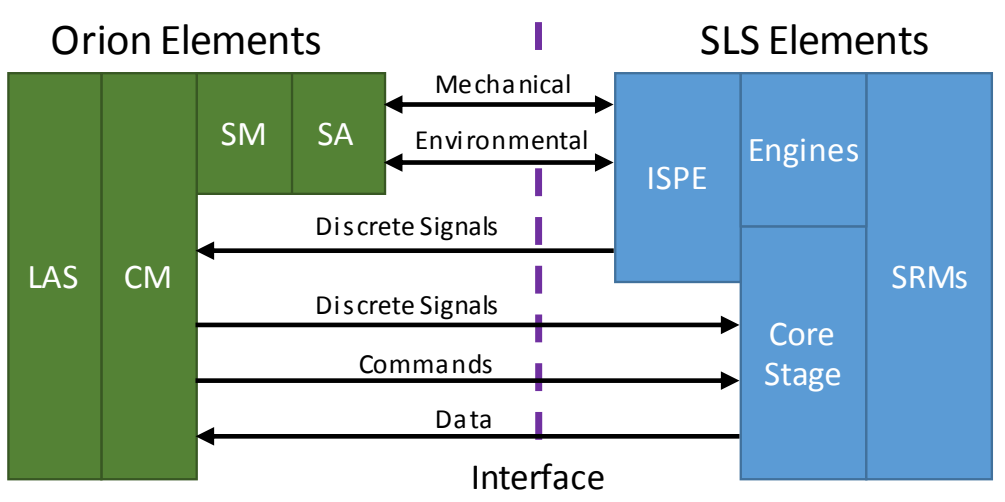

Figure 2. Orion to SLS Interface schematic, EM-1 configuration.

\section{B. Program Organization}

The organizational structure implemented by NASA to facilitate and expedite integration of the overall space transportation system, is discussed by Smith et al. ${ }^{7}$, and will only be revisited here quite briefly.

The NASA Headquarters Exploration Systems Development Directorate (ESD) manages the development, integration and eventual operation of the next generation space transportation capability. Supporting ESD, three programs were established to create the human-rated spacecraft, launch vehicle and associated ground infrastructure.

- The Orion MPCV Program is managed by Johnson Space Center (JSC) and is responsible for the performance, schedule, and budget that is allocated to this portion of the human spaceflight enterprise within NASA. It is accomplished with the help of a Prime Contractor, Lockheed Martin, and a substantial involvement of NASA engineering insight and oversight activities of a technical and programmatic nature.

- The SLS Program is managed by Marshall Space Flight Center (MSFC) with MSFC acting as the systems integrator. The hardware development is divided into five configuration items. There are NASA Element Offices providing insight to Prime contractors for the development of the core stage, the core stage engines, the Boosters (SRMs) and the ISPE. The Core Stage Flight Software is being developed in-house at MSFC.

- The Ground Systems Development Office (GSDO) is managed by Kennedy Space Center (KSC) and is charged with upgrading the ground processing and launch facilities to accommodate the SLS and Orion vehicles. 
NASA ESD has allocated program integration responsibilities to two internal organizations: the Cross-Program Systems Integration (CSI) Office, and the Programmatic and Strategic Integration (PSI) Office. CSI shares technical integration responsibilities with the Programs, including systems engineering, architectures, and safety. PSI focuses on programmatic integration, including cost, and schedule and risk.

To facilitate the technical integration, CSI instituted a Cross-program Integration Team (CPIT) and established six relevant Functional Areas (FA) to manage the effort. Within each FA, Integrated Task Teams (ITT), with designated co-leads from each Program, are formed to work specific aspects of the technical integration effort. In the Interfaces FA, an ITT exists for each Program to Program interface, including the Orion to SLS Interface, the SLS to GSDO interface, and the Orion to GSDO interface. The Orion to SLS ITT is further discussed in Section IV after first explaining the framework of the SECM.

\section{Systems Engineering Capability Model}

The Electronic Industries Alliance (EIA) publishes EIA 632, Processes for Engineering a System ${ }^{8}$, which is one of several industry standards that state "what to do" when defining and implementing systems engineering processes. As an accompaniment, EIA 731.1, Systems Engineering Capability Model, is a standard for establishing "how well" the processes are defined and implemented. Its purpose is to aid in the development and improvement of an organization's systems engineering capability.

The NASA policy for Systems Engineering" (NPR 7123.1) references EIA 632 as "a commercial document ... intended to provide a framework for developing and supporting universal SE discipline for both defense and commercial environments." The SECM comprises a hierarchical architecture of specific practices for each bottom tier process, and generic characteristics used to qualify the maturity and effectiveness of each process. The SECM architecture, shown in Figure 3, defines the breakdown structure and constituent components.

\section{A. Systems Engineering Domain}

The model organizes the breadth of the Systems Engineering Domain by defining Focus Areas, which subdivide into Themes and again into Specific Practices (SP).

The relevant breakdown path for this paper is:

- 1.0 Technical Category (not shown)

- 1.5 Integrate System Focus Area

- 1.5-2 Interface Coordination Theme

The Interface Coordination Theme is comprised of 8 Specific Practices. The scope of these practices include coordinating interface efforts, identifying technical baselines, and capturing and storing interface designs. (Note: These Specific Practices are not explicitly part of the argument made in the paper, and as such are not delineated here.)



Figure 3. SECM Architecture Structure and Components

\section{B. Capability Domain}

The other facet of the model (i.e. branch of the diagram in Figure 3) is the Capability Domain, which employs Generic Characteristics as criteria for establishing an organization's ability to perform Systems Engineering. As the name suggests, these characteristics are generic and therefore are to be applied to each Specific Practice to gauge process implementation maturity and effectiveness. The Generic Characteristics are subdivided into process related Generic Practices and non-process related Generic Attributes.

\section{Generic Practices}

EIA 731.1 defines Generic Practices to be used to enhance organizational continuous improvement. They are mutually exclusive but are grouped into capability levels, as shown in Table 1. Generic Practice Levels, and include: 
- Level 1 has no Generic Practices, implying that the process implementation is largely 'ad hoc'

- Level 2 has two Generic Practices that characterize a process developed for a specific instance (e.g. a project-developed process)

- Level 3 has four Generic Practices that characterize a process tailored from an organizational standard process

- Level 4 has two Generic Practices (augmenting the Level 3 practices) that characterize a process that employs metrics to track performance quantitatively and used to drive improvement.

- Level 5 has four Generic Practices that characterize a process driven by established efficiency and effectiveness targets based on business needs.

\section{Generic Attributes}

Generic Attributes are not aligned to maturity levels but rather are used to help organizations establish the impact of achieving a maturity level for a given process. This is accomplished via two basic questions: 1) what is the Effectiveness of the effort being expended? (Are the benefits worth the cost of the effort?), and 2) what is the Value of the products being generated? (Are the products beneficial to the intended recipients?)

\section{Level 3 Generic Practices}

For this paper, our argument is that the implementation of the Orion to SLS Interface Management process has achieved a generic capability Level 3 (Managed) via meeting the criteria established by the four Generic Practices (GP). More specifically, as taken from EIA 731.1, shown in Table 2:

Table 2. EIA 731.1 Level 3 Generic Practices

\begin{tabular}{|c|c|l|}
\hline Number & Informal Title & \multicolumn{1}{c|}{ Generic Practice } \\
\hline GP 3.1 & Establish a Standard Process & $\begin{array}{l}\text { Standardize and record a well-defined Focus Area process for the } \\
\text { organization that is designed to meet specific business goals, and is } \\
\text { based on experiences captured from previous programs. }\end{array}$ \\
\hline GP 3.2 & Tailor the Standard Process & $\begin{array}{l}\text { Tailor the organization's standard process using standard guidelines } \\
\text { to meet specific program or organizational needs }\end{array}$ \\
\hline GP 3.3 & Improve the Tailored Process & $\begin{array}{l}\text { Implement and improve the Focus Area activities (i.e., tailored } \\
\text { process) per established and approved formal procedures }\end{array}$ \\
\hline GP 3.4 & Improve the Standard Process & $\begin{array}{l}\text { Improve the organization's standard process using information from } \\
\text { work product reviews and process compliance reviews }\end{array}$ \\
\hline
\end{tabular}

It is important to note that this paper does not argue for achievement of the 'complete' level. As defined in EIA 731.1, "Being "at a level" means doing all of the practices (Generic and Specific) at that level in a manner consistent with the descriptions of the Generic Attributes at that level'. We have not attempted to map our NASA standard activities to the EIA 731.1 Specific Practices, and as such, an aspect of overall Level determination has not been addressed. Instead, our approach is to apply the Level 3 Generic Practices to the NASA standard activities, as a means of establishing a qualitative measure of continuous improvement for this process for the program and the institution.

\section{Level III Compliance Evidence}

This section provides the evidence that the SECM Level 3 Generic Practices of the previous section are met. The four Level 3 General Practices are addressed in separate subsequent subsections. Evidence is derived from our work products and experiences over the last 2 years of facilitating the Orion to SLS Interface ITT.

\section{A. GP 3.1 Establish a Standard Process}

- Standardize and record a well-defined Focus Area process for the organization that is designed to meet specific business goals, and is based on experiences captured from previous programs.

This section provides evidence that NASA has standardized and documented a well-defined Interface Management process at the institutional level that is aligned with the NASA business model, based on successful past program

American Institute of Aeronautics and Astronautics 
products and lessons learned, and is the mandatory basis for SE process implementation on all Space Programs across the Agency.

\section{NASA Systems Engineering Standard Processes}

As part of its ISO 9001 program (for quality management), NASA has established an Agency-level standard for Systems Engineering, in NPR 7123.1, NASA Procedural Requirements (NPR) for Systems Engineering ${ }^{10}$. The policy is framed around an SE "Engine" comprised of 17 processes, including Interface Management, as shown in Figure 4

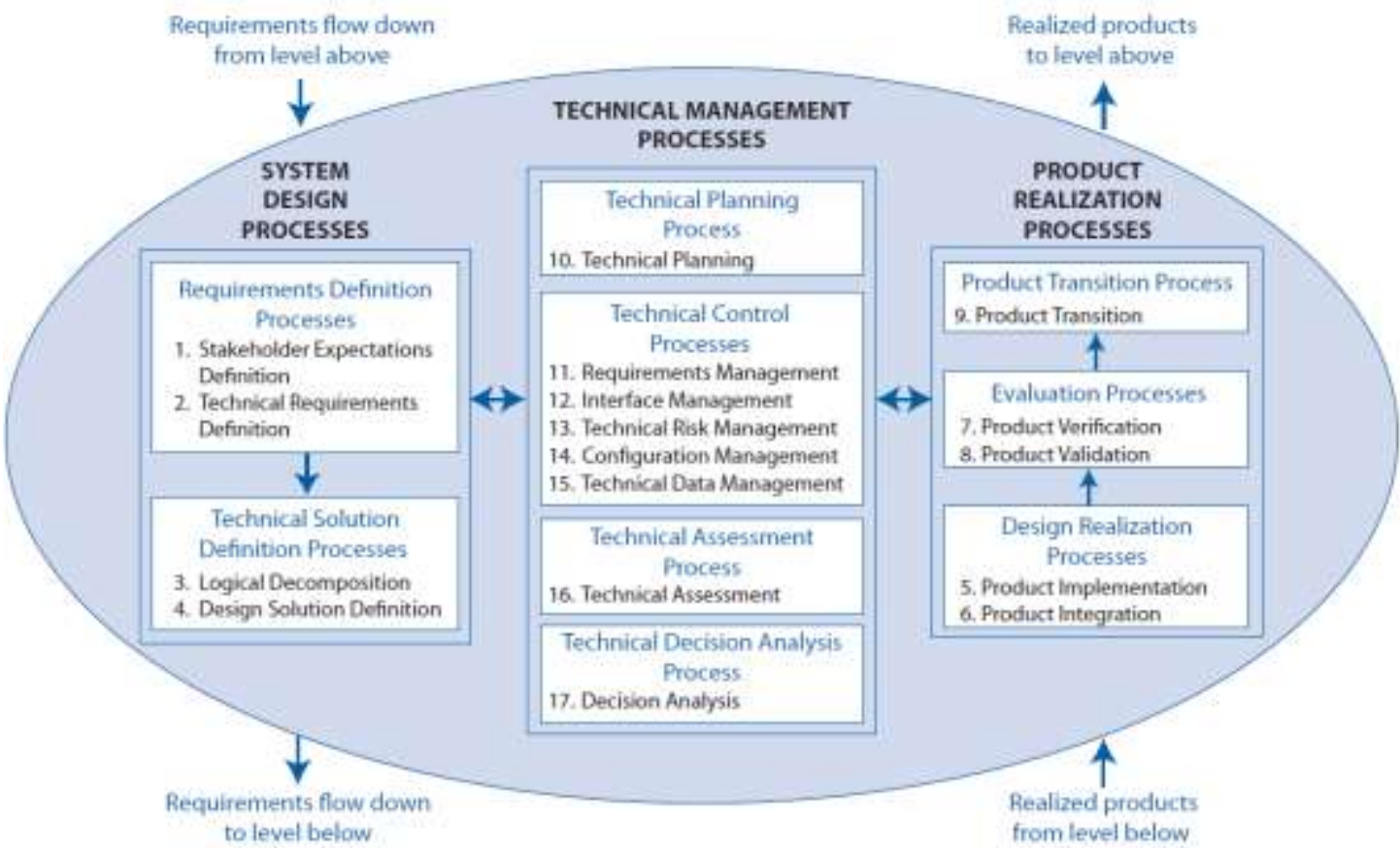

Figure 4. NASA Systems Engineering Engine.

per NPR 7123.1 (Ref 7), the process requirement for Interface Management is: "Center Directors or designees shall establish and maintain an Interface Management process to include activities, requirements, guidelines, and documentation for management of the interfaces defined and generated during the application of the system design processes." It can be seen that the NASA SE process requirements are maintained at a high level. This is because NASA performs SE on many different product types and program sizes, from human rated spacecraft, to heavy lift launch vehicles, to space station payloads, to interplanetary robotic (uncrewed) explorers. If the overarching requirements were more explicit, the cumulative tailoring effort to optimize these diverse instances would be extensive. However, the appendices of the NPR contain recommended practices in the form of flow diagrams with inputs, activities, and outputs for each process, which are commonly used as the starting point for tailoring. These flows are generally based on the "common denominator" activities for the majority of NASA programs. In addition, NPR 7123.1 provides Life Cycle Review and Milestone requirements, Program SEMP development requirements, and SE process tailoring requirements.

A useful supplement to NPR 7123.1 is the NASA Systems Engineering Handbook ${ }^{11}$ (NASA/SP-2007-6105 Rev.1), which provides additional guidance, generic outlines and typical checklists to aid programs in implementing their SE processes. Much of the data in this manual is derived from NASA and industry best practices and lessons learned, and is periodically updated. 


\section{Interface Management Process}

NASA 7123.1 (Ref. 10) further explains that the "interface management process is used to: (a) establish and use formal interface management to assist in controlling system product development efforts when the efforts are divided between Government programs, contractors, and/or geographically diverse technical teams within the same program and/or project; and (b) maintain interface definition and compliance among the end products and enabling products that compose the system, as well as with other systems with which the end products and enabling products must interoperate."

The 'typical' flow diagram for the Interface Management process, (cf. Figure 5), is discussed in both the NPR 7123.1 Appendices and NASA SP-6105.

The flow diagram illustrates five essential activities:

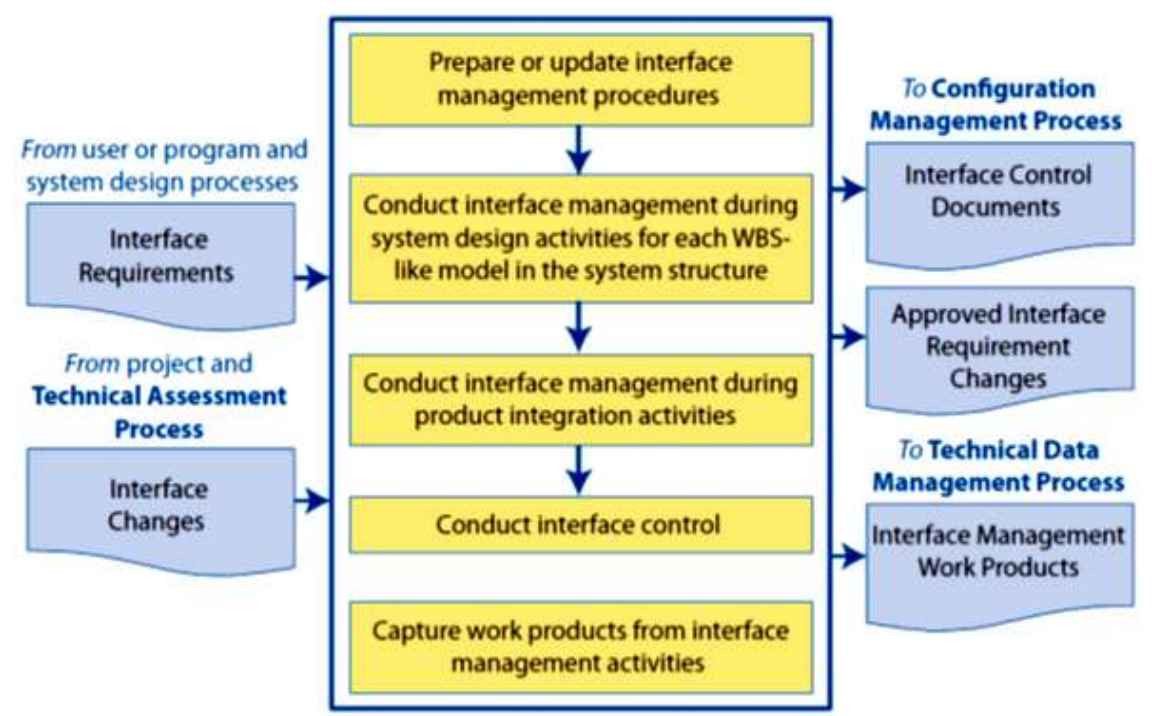

Figure 5. Interface Management Flow Diagram

- Prepare or update Interface Management procedures - this includes organizational structure and responsibilities, work flow, product development and control, and decision making

- Conduct Interface Management during System design activities - this includes coordination of the development of interface requirements and designs

- Conduct Interface Management during product integration activities - at the system to system level, physical integration for EM-1 is still 2 years away. However, this activity also includes verification planning, test planning, and assembly and checkout procedure development, which is underway.

- Conduct Interface Control - this includes maintaining the requirements and design agreements within the technical baseline, and using formal change management to make updates with control board approval

- Capture work products from Interface Management activities - this includes the archiving and dissemination of interface documents as well as working group minutes

The remaining Generic Practice subsections will show that we have tailored this process for Program use, improved the tailored process over time, and have plans to institute change to the institutional process database.

\section{B. Tailor the Standard Process}

- Tailor the organization's standard process using standard guidelines to meet specific program or organizational needs

This section provides evidence that the NASA ESD, with support of the Orion to SLS Interface ITT, had initially tailored the Interface Management standard process at the beginning of the programs to meet the needs of this level of integration.

It is important to clarify the use of the term "tailoring" in this paper in contrast to its usage in NPR 7123.1. Note that Ref. 7 states: "SE requirements tailoring is the process used to seek relief from SE NPR requirements consistent with program or project objectives, acceptable risk, and constraints." It also defines another term: "Customization is the modification of recommended SE practices that are used to accomplish the SE requirements." For this Orion to SLS effort, relief from the NPR requirements for Interface Management was not exercised. However, we have done significant customization of the recommended practices. Hence, the term 'tailoring', as used in this paper, and as understood to be for EIA 731.1, is meant to be synonymous with the NPR 7123.1 concept of 'customization.'

The ESD Directorate implementation of the SE NPR is ESD 10012, ESD Systems Engineering Management Plan, SEMP. ${ }^{12}$ An overarching framework and directions on individual ITT tailoring of the interface management process 
is given to mandate a consistent practice amongst the Orion, SLS, and GSDO programs. The initial Interface Management process tailoring addressed organization, workflow, products and control.

\section{Interface Management Organization}

As discussed in section II.B above, an ITT was initiated for each system to system interface. The Orion to SLS interface ITT is identified as the Orion-SLS Interface Working Group (OSIWG). A formal Charter ${ }^{13}$ delineates the OSIWG membership, roles, responsibilities and deliverables. It stipulates the following organizational aspects:

- The OSIWG is led by program-designated Orion and SLS working group co-chairs, acting as representatives of ESD as well as their respective programs.

- As the overall interface lead program, MPCV provides a designated Change Package Engineer (CPE) for each deliverable product.

- Technical discipline representatives from each program and programmatic representatives from ESD and the other programs comprise the remainder of the OSIWG membership.

- As working group topics and needs fluctuate, additional interface stakeholders may participate as necessary

\section{Interface Management Workflow}

The Orion to SLS Interface Management workflow is centered on product development. The Interface Requirements Document (IRD) is updated approximately every eight months, and typically delivered either ahead of a major milestone review (e.g. Critical Design Review), or as an outcome from a major review or design evolution. Since design follows specification, the Interface Control Documents (ICD) are typically updated approximately six months following the IRD.

The OSIWG meets regularly (usually weekly) to discuss topics related to content going into the next revision of the IRD or ICD. Preceding each meeting, a planning session among the OSIWG leadership is held to select topics, based on priority, for the upcoming OSIWG meeting. Presenters for the topics are identified. The presenters are responsible for preparing a presentation, which can be informational or decisional. The topics are announced in advance, via the standing meeting invite, to ensure OSIWG members and other pertinent stakeholders are aware of the specific topics and have opportunity to arrange for the meeting. Subsequent to each meeting, minutes are developed, containing discussion summaries, decisions made and new actions. These minutes provide a reference archive as well as disseminating information to those stakeholders unable to attend.

As time gets closer to update a document, a detailed schedule is developed showing all pertinent steps in the flow including: finalization of the proposed changes, submission date of the Change Request (CR), review period, comments due date, Technical Coordination Meeting (TCM) to disposition the comments, control board dates and finally document release. The weekly workflow is paced by this schedule.

\section{Interface Management Products}

The Orion-to-SLS Interface technical baseline is managed through an IRD and two ICDs. The respective roles and relationships among these documents is shown in Figure 6. The IRD holds the functional and performance requirements, and
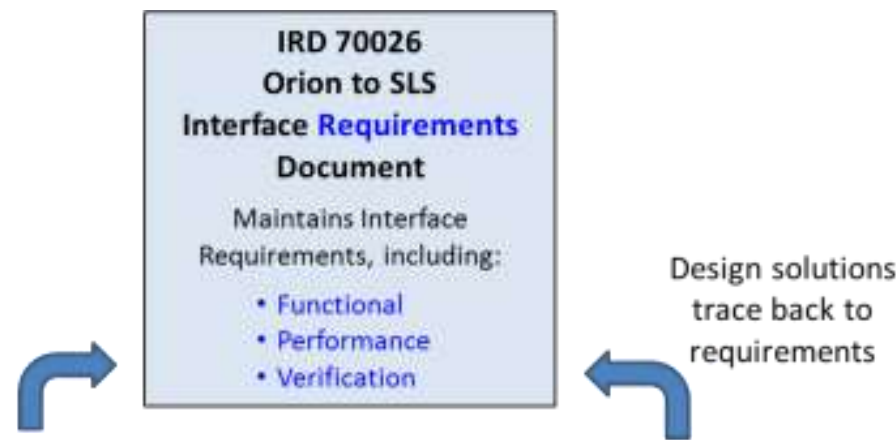

CEV-T-029800 Orion to SLS Hardware Interface Control Document

Maintains Physical Interface design agreements, including:

- Loads \& Structures

- Fluids and Gases

- Environments, Human Factors

- Electrical \& Communications

- Flight Performance

Figure 6. Orion to SLS IRD, and the Orion to SLS ICDs. 
the design solutions to those requirements are in the ICDs, in which the design features trace back to the specific invoking IRD requirements.

The IRD requirements cover the expected system interaction aspects: structural and thermal environments, mechanical and electrical connections, information and data exchange, and flight performance and safety constraints. Each requirement 'shall' statement is accompanied by a "rationale" statement intended to ensure that the requirement is interpreted in the proper context, including not only what it applies to but also where and when it applies.

The IRD was initially structured using a MPCV defined format and content template, derived from the NASA standard template guidelines. It was decided to segregate the ICD details into two volumes: Volume 1 is the Hardware ICD (including both mechanical and electrical hardware), and Volume 2 is the C\&DH ICD (including aspects for both SLS stages). Based on this, the initial ICD outlines were significantly tailored to accommodate the distributed documentation and serve the unique architectures of the Orion and SLS vehicles.

The IRD and ICD current baselines are maintained in the MPCV instantiation of CRADLE. CRADLE is a configuration controlled repository of the evolving technical baseline and offers complete end to end traceability of intra-program and inter-program technical specifications and how they are to be verified. The output of the IM process is captured in CRADLE, whether it is new requirements, how they are to be verified, or the associated mission effectivity. Versions of the documents are generated and posted on program controlled Wiki sites, to provide easy access. This tandem approach promotes and facilitates the use of this information across a wide group of stakeholders, particularly those under contract to the SLS and Orion programs.

\section{Interface Management Control}

Within the NASA management framework, the Program is typically the highest organizational level of integration. But in this case, each major system (the spacecraft, the launch vehicle and the ground infrastructure) was designated a Program, responsible for the development and operation of the given system. This necessitated the formation of a control board structure, shown in Figure 7, charged with the integration of these systems. this structure includes the Joint Integration Control Board (JICB), tri-chaired by the chief engineers from each program, charged with maintaining the cross program technical baseline, and the Joint Program Control Board (JPCB), tri-chaired by the respective program managers, responsible for the cross program budget and schedule baseline. These boards are the formal decision gates for revising the inter-system technical baseline, and as such, control the Orion-SLS interface requirements and design agreements.

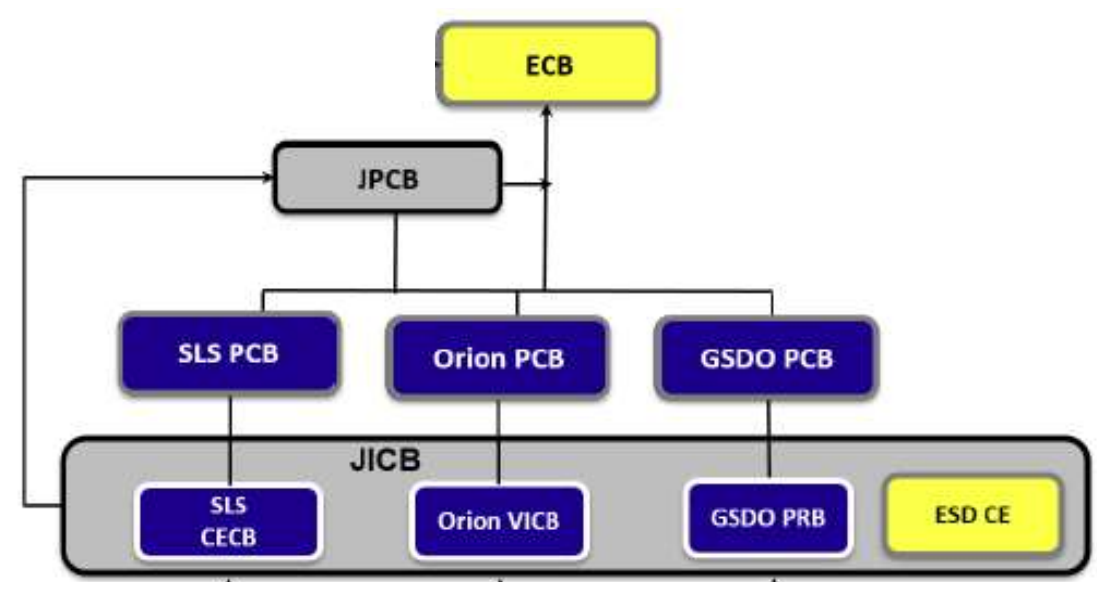

Figure 7. Cross program Control Board structure. (figure adapted from Ref. 7)

\section{Improve the Tailored Process}

- Implement and improve the [program] activities (i.e., tailored process) per established and approved formal procedures

This section provides evidence that the OSIWG has taken steps to improve the tailored process, addressing inefficiencies in the initial approaches as well as reacting to external decisions. The evidence is organized around the five Interface Management process steps shown in Figure 5. 


\section{Mandatory OSIWG Coordination - IM Procedure Improvement}

Within the NASA standard Interface Managing process, interface requirements are generated within the System Design process and then input to Interface Managing process. Initially, that is how the Programs operated. Discipline teams on either side identified interface requirements and proposed inclusion into the baseline via an engineering change request (CR) to the Orion to SLS IRD. Stakeholders would review the CR and provide comments. When proposed requirements had unintended technical, cost or schedule consequences for teams not involved with the CR submission, workflow times were significantly hampered by unnecessary iteration. In response, pre-coordination was adopted by various technical teams for significant proposed changes. With experience, it was clear that even expected small changes could have similar broad impacts and subsequent process impedance.

As a means to counter this pattern, the OSIWG modified the tailored process by requiring every proposed change to the IRD, large or small, be presented at an OSIWG forum, even those previously discussed and agreed to at other Cross-Program forums. This improvement has resulted in sizable cost and schedule avoidance, by allowing all interface stakeholders to have an opportunity to assess the change and reveal and address previously unidentified consequences before being submitted for a formal technical baseline change.

\section{ICD Working Groups and Technical Interchange Meetings - IM Procedure Improvement}

Once the IRD was initially baselined, and some attention was turning to ICD design implementation, the number of requested OSIWG topics started to grow. In addition, the implementation of the requirements in design space was necessitating additional layers of expertise (particularly implementing contractors) beyond the standing OSIWG membership. To address the ever increasing workload and participation, the OSIWG leadership decided to spawn two additional standing working groups, one to focus on the Hardware ICD and another on the C\&DH ICD. Each is led by the respective document's MPCV assigned Change Package Engineer (CPE), supported by a lead from the SLS program. Similar to the OSIWG, membership is largely unrestricted by organizational barriers and allows for topic dependent ebb and flow of participation. Continuity among the three working groups is facilitated by leadership and membership cross participation. Each forum is attended virtually from multiple sites/locations through internet-based telecommunication methods.

In addition, the OSIWG topics were not only becoming more numerous, but also more complex and far-reaching, to the extent that the discussions needed to enable decision making were becoming impractical via means of virtual communications (telecons, emails, etc.). Therefore the OSIWG arranged a formal face-to-face technical interchange meeting (TIM), making it more practical to address a large number of topics in all-day sessions with a wider group of stakeholders. The OSIWG typically holds two to three TIMs each year, where working group specialists reside to maximize the inclusion of affected parties. Many apparent impasses have been bridged through these TIMs, when prolonged impersonal telecom sessions have failed to achieve the desired results. These are also invaluable opportunities for otherwise unknown parties to develop personal relationships with counterparts in different companies and states, and be able to understand the other's needs and constraints.

American Institute of Aeronautics and Astronautics 


\section{Use of MBSE to Visualize Interactions - IM during Design improvement}

The OSIWG was struggling with communicating the various scenarios, in which interface requirements for offnominal conditions were applicable, to the integrated avionics testing communities in each program. It was decided to apply a Model Based Systems Engineering (MBSE) process $^{14}$ to develop a product that could aid in communicating the complexity to the testing teams as well as other stakeholders.

Using a variation of the IBM Harmony ${ }^{15}$ process, we initially identified integrated vehicle use cases for the off-nominal conditions involving system-to-system interaction. We then created white box activity diagrams for each use case, showing the activities for each system, how they were sequenced, and the conditional paths that could be taken. Finally, interface requirements were aligned to interaction activities to indicate how they are instantiated, as exemplified in Figure 8 (diagramming illustration only).

With these activity diagrams, we were able to define operational scenarios for each conditional path permutation, which was instrumental in working with the testing teams to define the test cases for each communication related interface requirement.

This improvement in working group communication effectiveness was so successful, we decided to develop additional activity diagrams for use cases gleaned from segments of the nominal mission profile with significant system-to-system interaction. These products yielded benefits in understanding timing issues, as well as highlighting changes in operational design

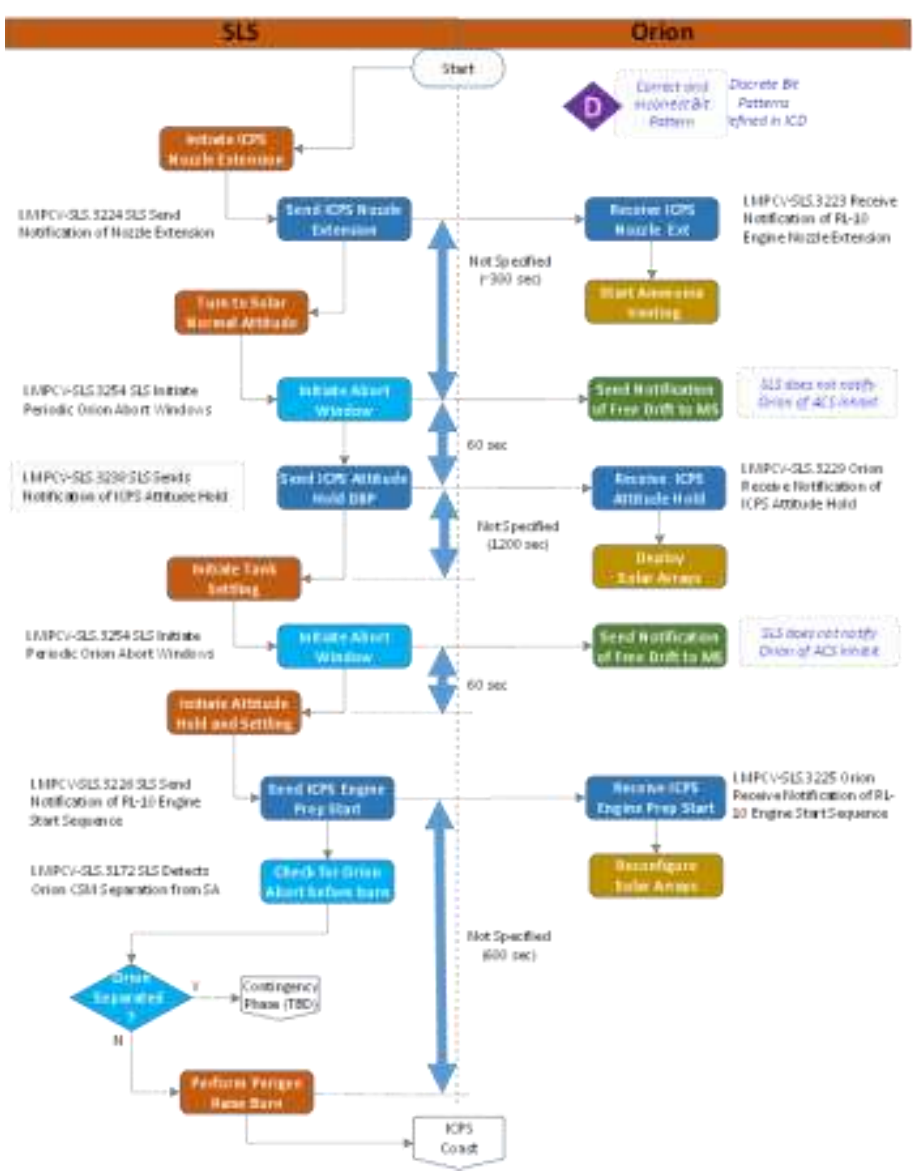

Figure 8. Example OSIWG developed Use Case Activity Diagram for the EM-1 flight.

The use of MBSE to develop use cases and activity diagrams is now a standard aspect of our Interface Management process. It is currently being used to develop operational concepts and eventual designs for future block upgrades to each system.

\section{Use BDEAL/BSHEALS for agreements - IM control improvement}

Since the interfaces are managed as bilateral Program to Program interface teams (with interface products), there is naturally a need to formally track data exchanges and hardware/software transmittals that are essential for systems design. While bilateral exchanges could be managed differently by each interface, the Orion to SLS interface team (and the others) are required to utilize an enterprise wide consistent approach as outlined by Smith et al. in Ref. 7. Hence, two databases are in place for any interface team, one for data (the Bilateral Data Exchange Agreements List, or BDEALS) and one for hardware/software (Bilateral Software/Hardware Exchange Agreements List, or BSHEALS). A key aspect of this IM tool is the use of the commitment date/schedule for delivering the items from one Program to the other. The establishment of the list is also controlled by the programmatic board structure discussed earlier. Commitments are negotiated between programs before establishing a new line item on either list, or when changes are needed a best effort renegotiation is carried out and updates are formally reported for management's insight and visibility.

American Institute of Aeronautics and Astronautics 
As designs evolved to the CDR level of maturity, the OSIWG encountered more and more situations where interface assessments relied upon the exchange of assumptions, data, and preliminary design analyses across opposite sides of the interface. Whereas the BDEALS and BSHEALS were well established for select hardware and software deliverables (finite element models of systems, mass properties estimates, for instance), it became necessary to augment the Agreements Lists with even more particular information pertinent to defining good interface requirements and ensuring their design implementation. Much of the requisite information was either generated by or requested by the implementing prime contractors for the ISPE EM-1 upper stage and the Orion MPCV crew module. Thus the OSIWG worked with the cross program Agreements team and increasingly leveraged this formal mechanism to identify, and implement, the key exchanges of technical material in support of the OSIWG ITT process and products. A case in point was the delivery of the Orion SM auxiliary thruster engines plume data for an SLS assessment of plume effects, and subsequently that an interface requirement can be added to restrict SM auxiliary thruster operations post-separation.

\section{Evolution of Requirements Verification Approach - IM during Integration Improvement}

In the early design phase, each Program (either Orion or SLS) was to be responsible for verification of respective system requirements, including the applicable interface requirements. Then, the testing community planned to build an Orion-SLS integrated hardware-in-the-loop test facility in Denver, Colorado. This Integrated Test Lab (ITL) would be used to conduct verification of system and C\&DH interface requirements. The test rig would include Flight Equivalent Units (FEU) of the Orion avionics and high-end emulators of the SLS avionics. Because of the paired nature of communication interface requirements (e.g. Orion shall send command X...; and, SLS shall receive command $\mathrm{X} . .$. ) it was reasonable to consider doing a single test to verify both the sending and receiving requirements.

To leverage this efficiency, the concept of Joint Verification was initiated. In the initial Joint Verification model, related $\mathrm{C} \& \mathrm{DH}$ interface requirements are paired, and a lead program is identified, who prepares the planning, conducts the testing, and generates the report. Both programs have approval of each step. The MPCV-SLS IRD Verification Cross Reference Matrix (VCRM) was modified to reflect this verification approach.

Later, it was realized that the SLS Emulator would be sufficient to exercise SLS Computer to Orion Computer interaction, but was not going to have the critical controller FEUs needed to completely verify some of the SLS requirements. To address this situation, the OSIWG developed the "Joint Analysis with Split Test" verification methodology. In this methodology, the same test case is run at both the Orion ITL and at the SLS System Integration lab (SIL) in Huntsville, Alabama. Then the lead program compares each test report to ensure the system interaction from the first motion on one side of the interface, across the interface, to the last motion on the other side of the interface, was consistent and as expected, in what we termed a Joint Analysis. This analysis was to be approved by both Programs.

The next evolutionary step came in trying to ensure that 'the same test' was indeed performed at each facility. The OSIWG developed the concept of 'common conditions,' which would be used by both Programs in the development of respective test requirements and procedures for the given interface requirements. After several iterations, the 'common conditions' were defined according to the features shown in Table 3.

\section{Table 3. Orion MPCV to SLS IRD Joint Verification Common Conditions}

\begin{tabular}{|c|l|}
\hline \multicolumn{1}{|c|}{ Features } & \multicolumn{1}{c|}{ Definitions } \\
\hline Common Situations & $\begin{array}{l}\text { Flight scenarios in which the function defined by the requirements pair is } \\
\text { exercised. Situations may be aligned individually or in combination to test cases }\end{array}$ \\
\hline Common Indicators & $\begin{array}{l}\text { Observable and measurable parameters that provide evidence of the cross system } \\
\text { response and the execution of the function defined by the requirements pair }\end{array}$ \\
\hline Common Outcomes & $\begin{array}{l}\text { The expected data and values for each of the common indicators, as predicted by } \\
\text { models and design. }\end{array}$ \\
\hline
\end{tabular}

It then became apparent that when the respective Programs implemented the common conditions, such that each program ran test cases to cover the common situations, collected the data associated with the common indicators, and that values of that data was as defined in the common outcomes, then the Joint Analysis effort was relatively trivial. By definition the tests would match. As such, we superseded the 'post processing' Joint Analysis, with the 'preprocessing' Common Conditions in this Joint Verification approach.

This evidence shows that the OSIWG was able to modify its interface requirements verification approach not just once, but several times to respond to external changes and incorporate self-generated improvements. 


\section{Dealing with SBU/Limited Rights - Capture IM Products Improvement}

An essential aspect of Program to Program work is the technical interchange among each Program's prime contractors. The exchange of design details sometimes incurs concerns about exposing third party (contractor) proprietary or ITAR-restricted information, and Limited Rights information. The term "Limited Rights" is used to connote particular restricted information owned by one of the Program contractors; notably per the government's terminology the Limited Rights data means "data, other than computer software, that embody trade secrets or are commercial or financial and confidential or privileged, to the extent that such data pertain to items, components, or processes developed at private expense, including minor modifications." Hence, any cross program group such as OSIWG must have a means to overcome these concerns.

The OSIWG interface products incorporated more restricted data as the designs evolved to a CDR level of maturity and hence it became increasingly important for this interface to manage such information. Further, if data from either Program's restricted data/documents was utilized in the ICDs, then it had to be maintained as restricted. The NASA ESD approach has been to provide and support Internet-based tools for use as data and information repositories, including documents, drawings, and meeting and decision records. Beside the ITAR-designated or otherwiserestricted material repositories, training is also mandated for OSIWG participants before they are given access to restricted information. The standard NASA policy and processes are complied with by the OSIWG ITT team so as to manage and control access; thus, no additional overhead is created that is specific to OSIWG. While there is a time lag that naturally develops for working with restricted information, the burden is considered acceptable for ensuring that the Programs' contractor's Limited Rights information is not breached. It also frees the OSIWG to insert as much necessary technical detail into the interface products as needed by both sides of the interface.

\section{Incorporation of Mission Effectivity - Capture IM Products Improvement}

SLS performance will be augmented over time with planned design block upgrades. The initial system acquisition strategy was to design the Block 1 configuration (for 70 metric tonne lift to LEO), and then build and fly those units for the first 2 missions, EM-1 and EM-2. The scope of the IRD and ICDs were aligned with that plan. Then a few years ago, NASA decided to fly one configuration of the ICPS on EM-1, then implement planned upgrades and fly a second ICPS configuration on EM-2. (Since the first flight is uncrewed, it was logical to forgo some human rating related modifications to the Delta IV-derived stage to realize sizable cost and schedule impact avoidance.) The resulting ICPS configurations turn out to be substantially different from an Orion interface standpoint.

The initial plan was to develop a new IRD and ICDs for each major block upgrade. We debated generating a new IRD for this unplanned mid-block configuration change. Instead, the decision was made to substantially customize the flight effectivity matrix into the IRD that would designate which configurations have effectivity for a given requirement. Some requirements were effective for both configurations, while others are effective only for EM-1, and still others only for the EM-2 mission flight.

The ICPS change also forced an assessment of requirements effectivity over the SLS vehicle flight phases. In some cases, interaction would be during Core Stage flight while others cases would be during upper stage (ISPE) flight. To address this need the flight effectivity matrix was expanded to indicate flight phase as well as configuration effectivity. This mapping effort in turn drove the realization that some requirements are also effective in the prelaunch phase, so this was added to the matrix as well, as shown in Figure 9. A significant byproduct was that this information was valuable to the system software developers and test planners.

\begin{tabular}{|c|c|c|c|c|c|c|c|c|}
\hline \multirow{2}{*}{$\begin{array}{c}\text { Document } \\
\text { Section }\end{array}$} & \multirow{2}{*}{$\begin{array}{c}\text { Requirement } \\
\text { Number }\end{array}$} & $\begin{array}{c}\text { Requirement } \\
\text { Title }\end{array}$ & \multicolumn{3}{|c|}{ EM-1 Configuration } & \multicolumn{3}{|c|}{ EM-2 Configuration } \\
\cline { 4 - 9 } & & $\begin{array}{c}\text { Prelaunch } \\
\text { Phase }\end{array}$ & $\begin{array}{c}\text { Core Stage } \\
\text { Flight }\end{array}$ & $\begin{array}{c}\text { ICPS } \\
\text { Flight }\end{array}$ & $\begin{array}{c}\text { Prelaunch } \\
\text { Phase }\end{array}$ & $\begin{array}{c}\text { Core Stage } \\
\text { Flight }\end{array}$ & $\begin{array}{c}\text { ICPS } \\
\text { Flight }\end{array}$ \\
\hline
\end{tabular}

Figure 9. Requirements Effectivity Matrix

Work is currently underway on the first major SLS block upgrade to 100 metric tonne lift to LEO. Based on the success of the IRD Effectivity Matrix in allowing interface requirements for sibling configurations to be specified in a single document, the decision was made to break from the original plan and maintain the IRD as a multiple configuration specification, via the Effectivity matrix. (At the time of this assessment, an analogous decision has not yet been made for the ICDs.)

American Institute of Aeronautics and Astronautics 


\section{Improve the Standard Process}

- Improve the organization's standard process using information from work product reviews and process compliance reviews

This section describes the steps taken by NASA to improve the standard Interface Management process, by incorporating lessons learned that can be beneficial for future programs. (Note that NASA does have a formal Lessons Learned Information System ${ }^{16}$ to facilitate and coordinate the dissemination of knowledge gained from prior work.)

To be fully compliant with EIA 731.1 Level 3 Generic Practices, the NASA standard process needs to be improved to incorporate the new lessons. This can be accomplished in several ways: Invoking change to the NPR 7123.1 Interface Management process requirements or description of the recommended process activities; Promoting enhancements to NASA SP-6105 guidance, outlines and checklists (Ref. 11); or, driving improvements to the respective NASA JSC and NASA MSFC adaptations of the Agency level SE policies and guidelines. (These NASA Center-level renderings provide approved tailoring to accommodate the relevant business models of the Centers).

The NPR 7123.1 requirements and recommended activities for Interface Management are maintained at a strategic level. It is our opinion that we have not performed any process improvements that are significant enough to propose a change to this aspect of the NASA standard. We do however believe that a few of our improvements should be captured in the accompanying guidance provided in NASA SP-6105. Guidance provided at the Center Level could be updated as well but this has not been investigated to date.

Our candidates for improvement to the standard process are discussed below.

\section{Specific Recommended Improvements}

- IM Procedure Improvements

- Incorporate into SP-6105, a discussion on mandatory pre-coordination of changes in advance of the $\mathrm{CR}$ as a best practice

- Incorporate into SP-6105, a discussion on the use of multiple forums to meet IM needs, as a best practice.

- IM Products Improvements

- Incorporate into SP-6105, a discussion on the use of Mission Effectivity matrices for hosting multiple configurations in single documents, as an available IM technique. Provide references and examples

- Incorporate into SP-6105, a discussion on dealing with SBU/Limited Rights data needing to be captured in the IM documentation, as a lessons learned.

- IM Design Improvements

- Incorporate into SP-6105, a discussion on the use of MBSE Activity Diagrams to model highly interactive interfaces in support of design and testing an available IM technique. Provide references and examples

- IM Integration Improvements

- Incorporate into SP-6105, a discussion on the use of Common Conditions for controlling distributed C\&DH interface testing as an available IM technique. Provide references and examples.

- IM Control Improvements

- Incorporate into SP-6105, a discussion on the use a Bilateral Exchange Agreements for managing and controlling exchange agreements, as a best practice. Provide references and examples for establishing a system.

\section{General Recommended Improvements}

- Include a discussion on the criticality of Working Group agility to Interface Management Success

The assessment incentivized us to go back through the numerous the modifications made to our organization, workflows and templates over the last few years. These were necessary to address changes in our customer needs, and working environment in addition to making adjustments for inefficiencies. Looking at the collective changes, it became apparent that the OSIWG had to be agile to make them all happen without any appreciable loss of productivity. In retrospect, we were willing to look at the new conditions, assess the options and select the best approach, even when the approach was met with understandable cultural resistance of "we've never done it that way". With backing from respective management, we were able to gain consensus and move forward. In addition, no aspect of our implementation was "off the table" for change. This was not a conscious decision at the time, but is apparent in 
retrospect when considering some of the innovative approaches we adopted. Our recommendation to others performing Interface Management would be to adopt these characteristics of an agile organization:

1) Keep the goal of efficient Interface Management as your prime focus,

2) Invoke changes as needed to remain responsive to your stakeholders, and,

3) Be willing to change any aspect of organization, workflow and products as necessary.

- Promote an alternative way to capture and disseminate Lessons Learned

NASA places a strong emphasis on Lessons Learned from its program's successes and failures. Agency and Center Level databases exist with numerous lessons learned from the past 50 years for use by engineers and managers to improve quality, efficiency and safety. They have proven to be very useful tools for NASA and Industry. The databases are typically organized by discipline and systems type, and are searchable by title and keywords. Nevertheless, it can be difficult to find a lesson that applies directly to a given current situation, especially when it involves process. The databases seem to naturally be oriented toward lessons associated with technology (e.g. engine testing, materials failures, and such). Finding a lesson on an aspect of something as esoteric as Interface Management can be more elusive.

This assessment, especially the consideration of the fourth practice - Improve the Standard Process, has given rise to a proposed strategic consideration of NASA: Augment the current lessons learned capture databases by capturing and disseminating applicable lessons learned by incorporating those lessons into NASA standards and guidelines. NASA standards for processes and other aspects are currently updated and improved under the auspices of the Office of Chief Engineer. However, the facet of using process continuous improvement as a mechanism for managing specifically lessons learned has not yet been formally instituted. For technology related lessons, the lessons learned database should continue to be the primary capture and dissemination tool. But for process related lessons, the standards, and particular the associated guidance, should be considered for lessons capture.

\section{Conclusion}

This paper summarizes the assessment of the Orion-SLS Interface Management process in achieving the EIA 731.1 Systems Engineering Capability Model Level 3 Generic Practices. Evidence was provided for each of the four relevant practices. Our contention is that sufficient evidence is available to demonstrate meeting the first three practices: Establish a Standard Process, Tailor the Standard Process, and Improve the Tailored Process. However, for the fourth practice: Improve the Standard Process, is partially complete since we have not yet submitted our proposed updates at the time of this writing. Assuming some or all recommendations will eventually be accepted, then once incorporated into the institutional process, we will have achieved the goal of GP Level 3 for Interface Management.

\section{Acknowledgments}

The authors would like to acknowledge the contributions of the wider stakeholder group of OSIWG members, which includes dozens of discipline and programmatic representatives from NASA Johnson Space Center, Marshall Space Flight Center, Glenn Research Center, including some located at the prime contractors for the SLS and Orion programs. The summary of this paper is a distillation of insights from several years of interface maturation activity among this wide group of stakeholders.

In particular, the authors acknowledge the sponsorship of the Orion and SLS technical leadership, and the guidance of the Cross Program Integration Team leadership, specifically, Dr. Leann Thomas, Mr. Jeff Spencer, Mr. Gary Langford, and Lisa Hammond, all of NASA.

American Institute of Aeronautics and Astronautics 


\section{References}

${ }^{1}$ Price, M., Early, J. M., Curran, R., Benard, E., and Raghunathan, S., “Identifying Interfaces in Engineering Systems," AIAA Journal, Vol. 44, No. 3, 2006, pp. 529-540.

${ }^{2}$ Systems Engineering Capability. Model. EIA-731.1. EIA-731.1. (Formerly EIA/IS-731.1), Electronic Industries Alliance, August 2002.

${ }^{3}$ Creech, S. D., "NASA's Space Launch System: An Enabling Capability for Discovery," IEEE Aerospace Conference 2014, Big Sky, Montana, March 1-7, 2014.

${ }^{4}$ Donahue, B. B., "The Space Launch System: Development Progress," AIAA SPACE 2016 Conference and Exposition, Long Beach, CA, September 2016.

${ }^{5}$ Marshall, P., and Norris, S. D., "Orion Program Status", AIAA SPACE 2013 Conference and Exposition, SPACE Conferences and Exposition, (AIAA 2013-5476).

${ }^{6}$ Norris, S. D., Marshall, P., Cichan, T., "Orion: lessons Learned from EFT-1 and EM-1, AA-2, and EM-2 status", AIAA SPACE 2016 Conference and Exposition, Long Beach, CA, September 2016.

${ }^{7}$ Smith, M., McConnaughey, P. K., Hill, W. C., Dan Dumbacher, D., and Pirtle, Z. T., "A Technical Integration Approach for NASA's Deep Space Human Exploration Programs," Paper IAC-14-B3.1, 65th International Astronautical Congress, Toronto, Canada, dated 23 July 2012.

8 “Processes for Engineering a System," EIA-632, Electronic Industries Alliance, September 2003.

9 "NASA Systems Engineering Processes and Requirements," NASA Procedural Requirements (NPR) 7123.1B, April $18,2013$.

10 "NASA Systems Engineering Processes and Requirements," NASA Procedural Requirements (NPR) 7123.1B, April 18, 2013.

11 "NASA Systems Engineering Handbook Revision 1," NASA Special Publication NASA/SP-2007-6105 Rev1, NASA December 2007.

12 "NASA Exploration Systems Development: Systems Engineering Management Plan," ESD 10007 Revision D, NASA HQ, May 2016.

13 "Task Agreement: Orion Multi-Purpose Crew Vehicle (MPCV) Program-To-Space Launch System (SLS) Program Interface Integration Task Team," NASA ESD Program-to-Program Interface Task Team (ITT) Charter Document, November 2015.

${ }^{14}$ Holladay, J., "MBSE through the Project Lifecycle," An MBSE Overview (Presentation) presented at 2016 Model Based Engineering Workshop at NASA's Goddard Space Flight Center, April 2016.

15 "Systems Engineering Best Practices with the Rational Solution for Systems and Software Engineering," Deskbook Release 3.1.2, Model-Based Systems Engineering with Rational Rhapsody and Rational Harmony for Systems Engineering, February 2011.

16 "NASA Lessons Learned Information Systems," NASA Procedural Requirements (NPR) 7120.6 (publicly available), January 22, 2010. 